\title{
Inhomogeneous quantum diffusion and decay of a meta-stable state
}

\author{
Pulak Kumar Ghosh, Debashis Barik and Deb Shankar Ray* \\ Indian Association for the Cultivation of Science, Jadavpur, Kolkata 700 032, India
}

\begin{abstract}
We consider the quantum stochastic dynamics of a system whose interaction with the reservoir is considered to be linear in bath co-ordinates but nonlinear in system co-ordinates. The role of the space-dependent friction and diffusion has been examined in the decay rate of a particle from a meta-stable well. We show how the decay rate can be hindered by inhomogeneous dissipation due nonlinear system-bath coupling strength.
\end{abstract}

\section{INTRODUCTION}

The system-reservoir model [1, 2, 3, 4, 5, 6] has been the standard paradigm for description of quantum dissipation [7] over the last few decades. Its application is ubiquitous in physics and chemistry. In the overwhelming majority of the situations the interaction between the system and reservoir is considered to be linear in bath co-ordinates and linear in system co-ordinates. This in turn relates the additive noise of the thermal bath with linear dissipation of the system through fluctuationdissipation relation. However when coupling between the reservoir is linear in bath co-ordinates but nonlinear in system coordinates, one encounters multiplicative noise 8, 9, 10, 11, 12, 13, 14, 15, 16, 17] and nonlinear dissipation in the form of co-ordinate dependent friction. The role of the space-dependent friction in classical context has been explored in several issues, e. g., charge transfer reaction in a polar medium and activated rate processes in the overdamped regime 18, 19, 20, 21, 22, fluctuationinduced transport 23, 24, stochastic resonance 26, 27], noise-induced transition [28, 29], etc. to name a few.

In the present paper we are concerned with spacedependent friction and multiplicative noise in a quantum mechanical context. A standard treatment of quantum dissipation based on linear interaction between the system and the reservoir was put forward in early eighties by Caldeira and Leggett [2, 3], which found extensions and wide applications in several areas of condensed matter and chemical physics 2, 3, 27, 25], particularly in quantum interference devices, polaron in a magnetic field, quantum decay processes etc. However, the exploration of physics of nonlinear system-reservoir coupling in a quantum system has been the subject of recent interest only. It has been observed that quantum dissipation can reduce the appearance of meta-stable state and barrier drift in a double-well potential 21]. Nonlinear coupling has been modeled by Tanimura and co-workers [5] in the study of elastic and inelastic relaxation mechanisms, and their interplay in the Raman and infrared spectra. A quantum transport as a consequence state-dependent diffusion has been investigated 17 in a periodic potential. A closer look into the nature of nonlinear coupling

\footnotetext{
*Email address: pcdsr@mahendra.iacs.res.in
}

(say, a linear in bath co-ordinate but quadratic in system co-ordinate) in contrast to linear coupling suggests that while the latter induces displacement (Fig.1(a)), the former coupling induces fluctuations of the system potential (Fig.1(b)). The effect of this fluctuation has been addressed in both spin-spin and spin-lattice relaxation processes [30, 31, 32, 33]. The fluctuation in the system potential due to nonlinear coupling, in general, tends to make the effective potential nonlocal in addition to making the diffusive process inhomogeneous in nature. This consideration is therefore of significant relevance in the context of quantum rate processes and it is worthwhile to examine the nature of the decay of a meta-stable state as a result of inhomogeneous quantum diffusion. Based on a coherent state representation of the noise operator and canonical thermal Wigner distribution 34] of the bath oscillators nonlinearly coupled to the system we have recently developed the quantum stochastic dynamics in the overdamped limit [17]. This has been carried out after adiabatic elimination of the momentum in the relevant Langevin dynamics which takes care of systematic quantum corrections to the potential.

Our aim in this paper is to search for the quantum signature of this nonlinear coupling on the decay rate, particularly in the pre-exponential factor and the generalized effective potential. We have emphasized how to take care of correct order of dissipation and appropriate thermodynamic consistency to avoid spurious term in the steady state distribution [9, 11] and show how the decay rate can be reduced by inhomogeneous dissipation.

The outlay of the paper is as follows: In Sec.II we introduce briefly the basic aspects of quantum stochastic dynamics with nonlinear coupling on a general setting. This is followed by a quantum Langevin equation in the overdamped limit. Sec.III is devoted to the calculation of decay rate from a meta-stable state under the influence of inhomogeneous diffusion and dissipation. This paper is concluded in Sec.IV.

\section{QUANTUM STOCHASTIC DYNAMICS WITH NON-LINEAR COUPLING}

We consider a particle of unit mass coupled to a medium comprised of a set of harmonic oscillators with frequency $\omega_{j}$. This is described by the following system- 
bath Hamiltonian [5, 21].

$$
\hat{H}=\frac{\hat{p}^{2}}{2}+V(\hat{q})+\sum_{j}\left[\frac{\hat{p}_{j}^{2}}{2}+\frac{1}{2}\left(\omega_{j} \hat{x}_{j}-\frac{c_{j}}{\omega_{j}} f(\hat{q})\right)^{2}\right]
$$

Here $\hat{q}$ and $\hat{p}$ are the coordinate and momentum operators of the particle and the $\left\{\hat{x}_{j}, \hat{p}_{j}\right\}$ are the set of coordinate and momentum operators for the bath oscillators with unit mass. The system particle is coupled to the bath oscillators nonlinearly through the general coupling terms $\frac{c_{j}}{\omega_{j}} f(\hat{q}) . c_{j}$ is the coupling strength. The classical counterpart 35] of the form (2.1) is known for many years and also the nonlinear coupling in quantum system has been studied in a few occasions [5, 21]. The potential $V(\hat{q})$ is due to external force field for the system particle. The coordinate and momentum operators follow the usual commutation relations $[\hat{q}, \hat{p}]=i \hbar$ and $\left[\hat{x}_{j}, \hat{p}_{k}\right]=i \hbar \delta_{j k}$.

Eliminating the bath degrees of freedom in the usual way we obtain the operator Langevin equation [6, 17] for the particle

$$
\begin{aligned}
\dot{\hat{q}}(t) & =\hat{p}(t) \\
\dot{\hat{p}}(t) & =-V^{\prime}(\hat{q}(t))-f^{\prime}(\hat{q}(t)) \int_{0}^{t} f^{\prime}\left(\hat{q}\left(t^{\prime}\right)\right) \gamma\left(t-t^{\prime}\right) \hat{p}\left(t^{\prime}\right) d t^{\prime} \\
& \quad+f^{\prime}(\hat{q}(t)) \hat{\eta}(t)
\end{aligned}
$$

where the noise operator $\hat{\eta}(t)$ and the memory kernel $\gamma(t)$ are given by

$$
\begin{aligned}
\hat{\eta}(t)= & \sum_{j}\left\{\frac{\omega_{j}^{2}}{c_{j}} \hat{x}_{j}(0)-f(\hat{q}(0))\right\} \frac{c_{j}^{2}}{\omega_{j}^{2}} \cos \omega_{j} t \\
& +\sum_{j} \frac{c_{j}}{\omega_{j}} \hat{p}_{j}(0) \sin \omega_{j} t
\end{aligned}
$$

and

$$
\gamma(t)=\sum_{j} \frac{c_{j}^{2}}{\omega_{j}^{2}} \cos \omega_{j} t
$$

It is clear from the operator Langevin equation Eq.(2.3) for the system that the noise operator is multiplicative and the dissipative term is nonlinear with respect to system coordinate due to the nonlinear coupling term in the system-bath Hamiltonian. In the case of linear coupling, i.e., $f(\hat{q})=\hat{q}$ Eq.(2.3) reduces to a quantum generalized Langevin equation [7] in which the noise term is additive and the dissipative term is linear.

In the Markovian limit the generalized quantum Langevin equation Eq. (2.3) reduces to the form

$$
\begin{aligned}
& \dot{\hat{q}}(t)= \hat{p}(t) \\
& \dot{\hat{p}}(t)=-V^{\prime}(\hat{q}(t))-\Gamma\left[f^{\prime}(\hat{q}(t))\right]^{2} \hat{p}(t) \\
& \quad+f^{\prime}(\hat{q}(t)) \hat{\eta}(t)
\end{aligned}
$$

where $\Gamma$ is dissipation constant in the Markovian limit.

Following Ref. 6] we then carry out a quantum mechanical average $\langle.$.$\rangle over the product separable bath modes$ with coherent states and system mode with an arbitrary state at $t=0$ in Eq. 2.6a and Eq. 2.6b to obtain a generalized Langevin equation as

$$
\begin{aligned}
\dot{q}= & p \\
\dot{p}= & -V^{\prime}(q)+Q_{V}-\Gamma\left[f^{\prime}(q)\right]^{2} p+Q_{1} \\
& +f^{\prime}(q) \eta(t)+Q_{2}
\end{aligned}
$$

where the quantum mechanical mean value of position and momentum operators $\langle\hat{q}\rangle=q$ and $\langle\hat{p}\rangle=p$. $Q_{V}=V^{\prime}(q)-\left\langle V^{\prime}(\hat{q})\right\rangle$ represent quantum correction due to nonlinearity of the system potential. $Q_{1}=$ $\Gamma\left[\left[f^{\prime}(q)\right]^{2} p-\left\langle\left[f^{\prime}(\hat{q})\right]^{2} \hat{p}\right\rangle\right]$ and $Q_{2}=\eta(t)\left[\left\langle f^{\prime}(\hat{q})\right\rangle-f^{\prime}(q)\right]$ are also quantum correction terms due to nonlinearity of the system-bath coupling function.

Furthermore quantum mechanical mean Langevin force is given by,

$$
\begin{aligned}
\eta(t)= & \sum_{j}\left\{\frac{\omega_{j}^{2}}{c_{j}}\left\langle\hat{x}_{j}(0)\right\rangle-\langle f(\hat{q}(0))\rangle\right\} \frac{c_{j}^{2}}{\omega_{j}^{2}} \cos \omega_{j} t \\
& +\sum_{j} \frac{c_{j}}{\omega_{j}}\left\langle\hat{p}_{j}(0)\right\rangle \sin \omega_{j} t
\end{aligned}
$$

To realize $\eta(t)$ as an effective c-number noise we now introduce the ansatz [6, 34, 37, 38, 39, 40] that the momentum $\left\langle\hat{p}_{j}(0)\right\rangle$ and the shifted coordinates $\left\{\frac{\omega_{j}^{2}}{c_{j}}\left\langle\hat{x}_{j}(0)\right\rangle-\right.$ $\langle f(\hat{q}(0))\rangle\}$ of the bath oscillators are distributed according to a Wigner canonical thermal distribution of Gaussian form as:

$$
\begin{gathered}
\mathcal{P}_{j}\left(\left\{\frac{\omega_{j}^{2}}{c_{j}}\left\langle\hat{x}_{j}(0)\right\rangle-\langle f(\hat{q}(0))\rangle\right\},\left\langle\hat{p}_{j}(0)\right\rangle\right) \\
=\mathcal{N} \exp \left\{-\frac{\left[\left\langle\hat{p}_{j}(0)\right\rangle^{2}+\frac{c_{j}^{2}}{\omega_{j}^{2}}\left\{\frac{\omega_{j}^{2}}{c_{j}}\left\langle\hat{x}_{j}(0)\right\rangle-\langle f(\hat{q}(0))\rangle\right\}^{2}\right]}{2 \hbar \omega_{j}\left(\bar{n}_{j}\left(\omega_{j}\right)+\frac{1}{2}\right)}\right\}
\end{gathered}
$$

so that for any quantum mechanical mean value, $\mathcal{O}_{j}\left(\left\{\frac{\omega_{j}^{2}}{c_{j}}\left\langle\hat{x}_{j}(0)\right\rangle-\langle f(\hat{q}(0))\rangle\right\},\left\langle\hat{p}_{j}(0)\right\rangle\right)$ which is a function of mean value of the bath operators $\left\langle\hat{x}_{j}(0)\right\rangle$ and $\left\langle\hat{p}_{j}(0)\right\rangle$, its statistical average $\langle\ldots\rangle_{S}$ is

$$
\left\langle\mathcal{O}_{j}\right\rangle_{S}=\int \mathcal{O}_{j} \mathcal{P}_{j} d\left\langle\hat{p}_{j}(0)\right\rangle d\left\{\frac{\omega_{j}^{2}}{c_{j}}\left\langle\hat{x}_{j}(0)\right\rangle-\langle f(\hat{q}(0))\rangle\right\}
$$

Here $\bar{n}_{j}\left(\omega_{j}\right)$ indicates the average thermal photon number of the $\mathrm{j}$-th oscillator at the temperature $T$ and $\bar{n}_{j}\left(\omega_{j}\right)=\left[\exp \left(\frac{\hbar \omega_{j}}{k_{B} T}\right)-1\right]^{-1}$ and $\mathcal{N}$ is the normalization constant.

The distribution $\mathcal{P}_{j}$ (Eq. (2.9) ) and the definition of statistical average Eq.(2.10) imply that c-number noise $\eta(t)$ must satisfy

$$
\langle\eta(t)\rangle_{S}=0
$$




$$
\left\langle\eta(t) \eta\left(t^{\prime}\right)\right\rangle_{S}=\frac{1}{2} \sum_{j} \frac{c_{j}^{2}}{\omega_{j}^{2}} \hbar \omega_{j}\left(\operatorname{coth} \frac{\hbar \omega_{j}}{2 k_{B} T}\right) \cos \omega_{j}\left(t-t^{\prime}\right)
$$

In the Markovian limit the noise correlation becomes 22 , 36]

$$
\begin{aligned}
\left\langle\eta(t) \eta\left(t^{\prime}\right)\right\rangle_{S} & =2 D_{0} \delta\left(t-t^{\prime}\right) \\
D_{0} & =\frac{1}{2} \Gamma \hbar \omega_{0}\left(\bar{n}\left(\omega_{0}\right)+\frac{1}{2}\right)
\end{aligned}
$$

where $\omega_{0}$ is the average bath frequency and the spectral density function is considered in the Ohmic limit. The use of Gaussian form [Eq. (2.9)] for a bath distribution needs some elaboration. It follows from a simple calculation of partition function of a nonlinearly coupled harmonic oscillators, that a Gaussian form of ansatz is not valid, in general. In the present case we emphasize that one is concerned here with a system-reservoir nonlinear coupling where the coupling is nonlinear in system coordinates but still linear in bath coordinates, so that the use of coherent state basis for harmonic oscillators enable us to factor out the dependence of nonlinear system coordinate in our calculation. This is also reflected in the fluctuation-dissipation relation (2.12) which retains its usual form, in spite of nonlinear system-reservoir interaction. Further to this we emphasize that the overdamped situation in our treatment refers to large $\Gamma$ rather than $\Gamma\left[f^{\prime}(x)\right]^{2}$. For a better validity of the theory the nonlinearity should not be too strong.

The equations (2.11) and (2.12) imply that the cnumber noise $\eta(t)$ is such that it is zero centered and satisfies the standard fluctuation-dissipation relation. We thus identify $\eta(t)$ as a classical looking noise with quantum mechanical content. The quantum Langevin equation can now be rewritten as follows:

$$
\begin{gathered}
\dot{q}=p \\
\dot{p}=-V^{\prime}(q)+Q_{V}-\Gamma\left[f^{\prime}(q)\right]^{2} p-2 \Gamma p f^{\prime}(q) Q_{f}-\Gamma p Q_{3} \\
-2 \Gamma f^{\prime}(q) Q_{4}-\Gamma Q_{5}+f^{\prime}(q) \eta(t)+Q_{f} \eta(t)
\end{gathered}
$$

The above equation is characterized by a classical force term, $V^{\prime}$, as well as its correction $Q_{V}$. The terms containing $\Gamma$ are nonlinear dissipative terms where $Q_{f}, Q_{3}$, $Q_{4}$ and $Q_{5}$ are due to associated quantum contribution in addition to classical nonlinear dissipative term $\Gamma\left[f^{\prime}(q)\right]^{2} p$. The explicit expressions for $Q_{V}, Q_{f}, Q_{3}, Q_{4}$ and $Q_{5}$ are given in the Appendix-A. The last term in the above equation contains a quantum multiplicative noise term in addition to the usual classical contribution $f^{\prime}(q) \eta(t)$. The classical limit of the above equation was derived earlier by Lindenberg and Seshadri 35]. Furthermore quantum dispersions due to potential and coupling terms in the Hamiltonian are entangled with nonlinearity. The quantum noise due heat bath on the other hand is expressed in terms of the fluctuation-dissipation relation.
While considering hydrodynamic interaction, i.e., when the fluctuation is position/state dependent or equivalently when the noise is multiplicative with respect to system variables the conventional adiabatic reduction of fast variables does not work correctly. To obtain a correct equilibrium distribution Sancho et al [9] had proposed an alternative approach to Langevin equation in the case of multiplicative noise system. By carrying out a systematic expansion of the relevant variables in powers of $\Gamma^{-1}$ and neglecting terms smaller than $O\left(\Gamma^{-1}\right)$ they obtained a Langevin equation corresponding to a FokkerPlanck equation in position space. This description leads to the correct stationary probability distribution of the system with coordinate-dependent friction.

We follow the same procedure in the quantum mechanical context. In this limit the transient correction terms $Q_{4}$ and $Q_{5}$ do not affect the dynamics of the position which varies in a much slower time scale. So the quantum Langevin equation Eq.(2.14) and Eq.(2.15) can be written as, respectively,

$$
\begin{aligned}
& \dot{q}=p \\
& \dot{p}=-V^{\prime}(q)+Q_{V}-\Gamma h(q) p+g(q) \eta(t)
\end{aligned}
$$

where

$$
\begin{aligned}
& h(q)=\left[f^{\prime}(q)\right]^{2}+2 f^{\prime}(q) Q_{f}+Q_{3} \\
& g(q)=f^{\prime}(q)+Q_{f}
\end{aligned}
$$

The variable $g(q)$ arises due to nonlinearity of the systembath coupling function $f(q), Q_{f}$ being the quantum correction to the classical contribution $f^{\prime}(q)$. For a linear coupling function $g(q)$ reduces to a constant.

Following the method of Sancho et al [9] further we obtain the Fokker-Planck equation in position space corresponding to Langevin equation Eq.(2.17)

$$
\begin{aligned}
\frac{\partial P(q, t)}{\partial t} & =\frac{\partial}{\partial q}\left[\frac{V^{\prime}(q)-Q_{V}}{\Gamma h(q)}\right] P(q, t) \\
& +D_{0} \frac{\partial}{\partial q}\left[\frac{1}{\Gamma(h(q))^{2}} g^{\prime}(q) g(q)\right] P(q, t) \\
& +D_{0} \frac{\partial}{\partial q}\left[\frac{g(q)}{\Gamma h(q)} \frac{\partial}{\partial q} \frac{g(q)}{\Gamma h(q)}\right] P(q, t)
\end{aligned}
$$

The Stratonovich prescription leads to corresponding Langevin equation as given by

$$
\dot{q}=-\frac{V^{\prime}(q)-Q_{V}}{\Gamma h(q)}-D_{0} \frac{g^{\prime}(q) g(q)}{\Gamma(h(q))^{2}}+\frac{g(q)}{\Gamma h(q)} \eta(t)
$$

Eq. (2.21) is quantum Langevin equation for multiplicative noise with position dependent friction in the overdamped limit (i.e., corrected upto $O(1 / \Gamma)$ ).

In the classical limit, i.e., $\hbar \omega_{0} \ll k_{B} T, h(q)=\left[f^{\prime}(q)\right]^{2}$, $g(q)=f^{\prime}(q), Q_{V}=0$ and $D_{0}=\Gamma k_{B} T$ so the quantum 
Langevin equation reduces to its classical counterpart as derived by Sancho et al [9]

$$
\dot{q}=\frac{1}{\Gamma\left[f^{\prime}(q)\right]^{2}}\left[-V^{\prime}(q)-\Gamma k_{B} T \frac{f^{\prime \prime}(q)}{f^{\prime}(q)}+f^{\prime}(q) \eta(t)\right]
$$

\section{DECAY RATE FROM A METASTABLE STATE}

Attention is now restricted here to the problem of barrier crossing dynamics in the presence of nonlinear system-bath coupling. The particle coordinate $q$ (which in our case is the quantum mechanical mean position) corresponds to the reaction coordinate and its values at the minima of $V(q)$ denote the reactant and product states separated by a finite barrier, the top being a metastable state representing the transition state. We choose the potential and the coupling function of the following forms ;

$$
\begin{aligned}
V(q) & =\frac{1}{2} a q^{2}-\frac{1}{3} b q^{3} \\
f(q) & =b_{1} q+\frac{1}{2} b_{2} q^{2}
\end{aligned}
$$

$a$ and $b$ are the potential parameters and the maximum and the minimum of the potential are at the point $q=$ $q_{b}=a / b$ and $q=q_{a}=0$, respectively. To clarify the role of the nonlinear system-bath coupling we now turn to Eq. (2.1), in which system-bath interaction is given by $\left(\omega_{j}\right.$ is absorbed in $\left.c_{j}\right)$,

$$
\begin{aligned}
\hat{H}_{i n t} & =-\sum c_{j} \hat{x}_{j} f(\hat{q}) \\
& =-\left(c_{1} \hat{x}_{1}+c_{2} \hat{x}_{2}+\ldots\right)\left(b_{1} \hat{q}+\frac{1}{2} b_{2} \hat{q}^{2}\right)
\end{aligned}
$$

The linear-linear coupling term $\left(\sum c_{j} b_{1} \hat{q}\right)$ is proportional to $b_{1}$ while square-linear coupling term $\left(\sum c_{j} b_{2} \hat{q}^{2}\right)$ is proportional to $b_{2}$. The linear-linear coupling model a very commonly used mechanism for studying quantum dissipative dynamics, e. g., energy dissipation from vibrational system mode to the heat bath modes during population decay. In addition to energy dissipation due to linear-linear coupling, one also encounters fluctuation in the system potential due to nonlinear coupling. We refer to Fig.1(b) where a schematic illustration of the fluctuation of system-bath coupling in a relevant potential well is presented. Since this fluctuation is the key mechanism for state-dependent diffusion, it is apparent that the calculation of transition rate in presence of statedependent diffusion differs from the calculation of escape rate for particles subject to thermally uniform noise, in that we may conveniently substitute the role of potential $V(q)$ by a generalized potential $\Psi(q)$ corresponding to the former case. A simple way to determine transition rate is to consider a steady state condition. The FokkerPlanck equation Eq.(2.20) in the overdamped limit can be rewritten in a more compact form as

$$
\frac{\partial P(q, t)}{\partial t}=\frac{\partial}{\partial q} \frac{1}{\Gamma h(q)}\left[V^{\prime}(q)-Q_{V}+\frac{D_{0}}{\Gamma} \frac{\partial}{\partial q} \frac{g(q)^{2}}{h(q)}\right] P(q, t)
$$

In the overdamped limit the stationary current from Eq.(3.4) is given by

$$
J=-\frac{1}{\Gamma h(q)}\left[V^{\prime}(q)-Q_{V}+\frac{D_{0}}{\Gamma} \frac{d}{d q}\left(\frac{g(q)^{2}}{h(q)}\right)\right] P_{s t}(q)
$$

The Eq. (3.5) can be rearranged into the following form,

$$
\frac{d}{d q}\left\{P_{s t} L(q) \exp \left[\frac{\Psi(q)}{D_{q}}\right]\right\}=-\frac{J \Gamma}{D_{q}} h(q) \exp \left[\frac{\Psi(q)}{D_{q}}\right]
$$

where

$$
\Psi(q)=\int^{q} \frac{V^{\prime}\left(q^{\prime}\right)-Q_{V}}{L\left(q^{\prime}\right)} d q^{\prime} ; \quad L(q)=\frac{g(q)^{2}}{h(q)}
$$

and $D_{q}=\frac{D_{0}}{\Gamma}$. To calculate a steady state solution we assume an absorbing boundary at a coordinate $q_{s}$ past the intervening potential maximum at $q=q_{b}$. So integrating Eq.(3.6) between $q=q_{a}$ and $q=q_{s}$, we have

$$
J=\frac{P_{s t}\left(q_{a}\right) L\left(q_{a}\right) \exp \left[\frac{\Psi\left(q_{a}\right)}{D_{q}}\right]}{\int_{q_{a}}^{q_{s}} \frac{\Gamma}{D_{q}} h(q) \exp \left[\frac{\Psi(q)}{D_{q}}\right] d q}
$$

The steady escape rate over the top of the potential is given by flux over population,

$$
k=\frac{J}{n_{a}}
$$

where $n_{a}$ is the population around the potential minimum $\left(q_{a}\right)$. The zero current defines an equilibrium condition in the neighborhood of $q_{a}$. Thus with $J=0$, probability distribution function near the potential minimum is given by

$$
P_{s t}(q)=P_{s t}\left(q_{a}\right) \frac{L\left(q_{a}\right)}{L(q)} \exp \left[-\int_{q_{a}}^{q} d q^{\prime} \frac{V^{\prime}\left(q^{\prime}\right)-Q_{V}}{L\left(q^{\prime}\right)} \frac{\Gamma}{D_{0}}\right]
$$

In terms of the generalized potential Eq. 3.10) can be rewritten as

$$
P_{s t}(q)=P_{s t}\left(q_{a}\right) \frac{L\left(q_{a}\right)}{L(q)} \exp \left[\frac{-\Psi(q)}{D_{q}}+\frac{\Psi\left(q_{a}\right)}{D_{q}}\right]
$$

Population around the bottom of the potential is given by

$$
\begin{aligned}
n_{a} & =\int_{q_{1}}^{q_{2}} P_{s t}(q) d q \\
& =P_{s t}\left(q_{a}\right) L\left(q_{a}\right) \exp \left[\frac{\Psi\left(q_{a}\right)}{D_{q}}\right] \int_{q_{1}}^{q_{2}} \frac{1}{L(q)} \exp \left[\frac{-\Psi(q)}{D_{q}}\right]
\end{aligned}
$$


where $q_{1}$ and $q_{2}$ are two points around potential minimum. So the expression for transition rate is given by

$$
k=\frac{D_{q}}{\Gamma} \frac{1}{\int_{q_{s}}^{q_{a}} d q h(q) \exp \left[\frac{\Psi(q)}{D_{q}}\right]} \frac{1}{\int_{q_{1}}^{q_{2}} d q \frac{1}{L(q)} \exp \left[\frac{-\Psi(q)}{D_{q}}\right]}
$$

By linearizing the generalized potential around maximum and minimum and evaluating the integrals in the usual way we obtain

$k=\frac{\left|\Psi^{\prime \prime}\left(q_{a}\right)\right|^{\frac{1}{2}}\left|\Psi^{\prime \prime}\left(q_{b}\right)\right|^{\frac{1}{2}} L\left(q_{a}\right)}{2 \pi \Gamma h\left(q_{b}\right)} \exp \left[-\left\{\frac{\Psi\left(q_{b}\right)-\Psi\left(q_{a}\right)}{D_{q}}\right\}\right]$

$\Psi(q)$ can be simplified further to yield

$$
\begin{aligned}
\Psi(q) & =\int^{q} \frac{V^{\prime}\left(q^{\prime}\right)-Q_{V}}{L\left(q^{\prime}\right)} d q^{\prime} \\
& =\int^{q} d q^{\prime}\left(V^{\prime}\left(q^{\prime}\right)-Q_{V}\right)\left(1+\frac{Q_{3}-Q_{f}^{2}}{\left(f^{\prime}\left(q^{\prime}\right)+Q_{f}\right)^{2}}\right)
\end{aligned}
$$

The rate constant (3.14) then reduces to the following expression,

$$
\begin{aligned}
k= & \frac{\sqrt{\left[\omega_{a}^{2}\left(1+\Delta_{1}\right)-\Delta_{v_{1}}\right]\left[\omega_{b}^{2}\left(1+\Delta_{2}\right)-\Delta_{v_{2}}\right]}}{2 \pi \Gamma h\left(q_{b}\right)\left(1+\Delta_{1}\right)} \\
& \times \exp \left[-\left\{\frac{\Psi\left(q_{b}\right)-\Psi\left(q_{a}\right)}{D_{q}}\right\}\right]
\end{aligned}
$$

where $\omega_{a}=V^{\prime \prime}\left(q_{a}\right)$ and $\omega_{b}=V^{\prime \prime}\left(q_{b}\right)$ are the frequencies at the bottom and barrier top, respectively. $\Delta_{1}, \Delta_{2}, \Delta_{v_{1}}$ and $\Delta_{v_{2}}$ are the contributions due to quantum correction and are given by,

$$
\begin{gathered}
\Delta_{1}=\left[\frac{Q_{3}-Q_{f}^{2}}{\left(f^{\prime}(q)+Q_{f}\right)^{2}}\right]_{q=q_{a}} \Delta_{2}=\left[\frac{Q_{3}-Q_{f}^{2}}{\left(f^{\prime}(q)+Q_{f}\right)^{2}}\right]_{q=q_{b}} \\
\Delta_{v_{1}}=\left[\frac{d}{d q}\left\{Q_{V}\left(1+\frac{Q_{3}-Q_{f}^{2}}{\left(f^{\prime}(q)+Q_{f}\right)^{2}}\right)\right\}\right]_{q=q_{a}} \\
\Delta_{v_{2}}=\left[\frac{d}{d q}\left\{Q_{V}\left(1+\frac{Q_{3}-Q_{f}^{2}}{\left(f^{\prime}(q)+Q_{f}\right)^{2}}\right)\right\}\right]_{q=q_{b}}
\end{gathered}
$$

The quantum nature of the transition rate (3.16) is manifested through the quantum correction due to nonlinearity of the potential and coupling function and the quantum inhomogeneous diffusion. It also contains the signature of nonlinear coupling in the pre-exponential factor and in the generalized potential $\Psi(q)$. More generally, the effect of state-dependent diffusion makes its presence felt in the dynamics of decay.

In order to check some inherent consistency of the rate expression we now examine the following situations. (a) First consider that the coupling function is linear, i. e., $b_{2}=0$ and $f(q)=q\left(b_{1}\right.$ is assumed to be unity). Then the generalized potential is given by

$$
\Psi(q)=\int^{q} d q\left(V^{\prime}\left(q^{\prime}\right)-Q_{V}\right)
$$

and the transition rate reduces to

$$
\begin{aligned}
k= & \frac{\sqrt{\left[\omega_{a}^{2}-\Delta_{v_{1}}\right]\left[\omega_{b}^{2}-\Delta_{v_{2}}\right]}}{2 \pi \Gamma} \exp \left[-\left\{\frac{\Delta \phi}{D_{q}}\right\}\right] \\
& \times \exp \left[-\left\{\frac{V\left(q_{b}\right)-V\left(q_{a}\right)}{D_{q}}\right\}\right]
\end{aligned}
$$

where

$$
\Delta \phi=\int_{q_{a}}^{q_{b}} \frac{Q_{V}(q)}{D_{q}} d q
$$

This quantify the characteristic quantum decay rate from a meta-stable state for particles subject to thermally uniform noise. On the other hand as the classical limit is approached all the quantum correction terms $\left(\Delta_{v_{1}}, \Delta_{v_{2}}\right.$ and $\Delta \phi$ ) due to nonlinearity of the potential tend to vanish and the quantum coefficient $\left(D_{q}\right)$ gets replaced by the classical one $\left(D_{q} \rightarrow k_{b} T\right)$. The transition rate (3.20) reduces exactly to Kramers rate in the overdamped limit.

$$
k=\frac{\omega_{a} \omega_{b}}{2 \pi \Gamma} \exp \left[-\left\{\frac{V\left(q_{b}\right)-V\left(q_{a}\right)}{k_{b} T}\right\}\right]
$$

(b) In order to check the thermodynamic consistency we consider further a symmetric periodic potential with periodicity $2 \pi$, i.e., $V(q)=V(q+2 \pi)$ and periodic derivative of coupling function with the same periodicity as that of the potential, i.e., $f^{\prime}(q)=f^{\prime}(q+2 \pi)$. Such a potential had been subject of interest in the problem of classical [23] and quantum transport [17] in inhomogeneous media.

Since the potential is periodic, $Q_{V}$ is also a periodic function as $Q_{V}=V(q)-\langle V(\hat{q})\rangle$. Similarly $Q_{f}$ is also periodic as $Q_{f}=\left\langle f^{\prime}(\hat{q})\right\rangle-f^{\prime}(q)$, and also $Q_{3}+2 f^{\prime}(q) Q_{f}$ is also periodic since $Q_{3}+2 f^{\prime}(q) Q_{f}=\left\langle\left[f^{\prime}(\hat{q})\right]^{2}\right\rangle-\left[f^{\prime}(q)\right]^{2}$. So from Eqs. (2.18) and (2.19) it is clear that $h(q)$ and $g(q)$ are also periodic functions of $q$ with periodicity $2 \pi$. Now it is easy to check that $\Psi(q)$ is also a periodic function with periodicity $2 \pi$.

$$
\Psi(q)=\Psi(q+2 \pi)
$$

Consider the bottom of the potential well at $q_{a}=2 \pi n$ and potential maxima at $q_{b}=(2 n+1) \pi$. Then with the help of Eq.(3.16) we find a transition rate from valley $n$ to valley $n+1$

$$
\begin{aligned}
k_{n+1, n}= & \frac{\sqrt{\left[\omega_{a}^{2}\left(1+\Delta_{1}\right)-\Delta_{v_{1}}\right]\left[\omega_{b}^{2}\left(1+\Delta_{2}\right)-\Delta_{v_{2}}\right]}}{2 \pi \Gamma h\left(q_{b}\right)\left(1+\Delta_{1}\right)} \\
& \times \exp \left[-\left\{\frac{\Psi\left(q_{b}\right)-\Psi\left(q_{a}\right)}{D_{q}}\right\}\right]
\end{aligned}
$$


Repeating these considerations for the transition rate from valley $n+1$ to valley $n$ yields

$$
\begin{aligned}
k_{n, n+1}= & \frac{\sqrt{\left[\omega_{a}^{2}\left(1+\Delta_{1}\right)-\Delta_{v_{1}}\right]\left[\omega_{b}^{2}\left(1+\Delta_{2}\right)-\Delta_{v_{2}}\right]}}{2 \pi \Gamma h\left(q_{b}\right)\left(1+\Delta_{1}\right)} \\
& \times \exp \left[-\left\{\frac{\Psi\left(q_{b}\right)-\Psi\left(q_{a}+2 \pi\right)}{D_{q}}\right\}\right]
\end{aligned}
$$

Thus the average particle velocity to a preferential direction is given by

$$
\begin{aligned}
\left\langle\frac{d q}{d t}\right\rangle & =2 \pi\left(k_{n+1, n}-k_{n, n+1}\right) \\
& =2 \pi k_{n+1, n}\left(1-\exp \left[-\left\{\frac{\Psi\left(q_{a}\right)-\Psi\left(q_{a}+2 \pi\right)}{D_{q}}\right\}\right]\right) \\
& =0
\end{aligned}
$$

We thus conclude that there is no occurrence of current for a periodic potential and periodic derivative of coupling with same periodicity. At the macroscopic level this confirms that there is no generation of perpetual motion of second kind, i.e., no violation of second law of thermodynamics. Therefore the thermodynamic consistency based on symmetry considerations ensures the validity of the present formalism.

Before we proceed to numerical results it is important to clarify the issues regarding the calculation of quantum correction terms. The details of the calculations of quantum correction terms are shown in the Appendix-A. One can calculate the value of quantum dispersion terms $\left\langle\delta \hat{q}^{n}\right\rangle$ by direct numerical simulation of the coupled Eqs. (A10) subject to appropriate boundary conditions. It is also instructive to deal with the quantum correction terms in the analytical way to find out the approximate value of quantum dispersion terms. For overdamped limit we neglect the $\delta \dot{\hat{p}}$ term from Eq. A9 to obtain

$$
\begin{aligned}
\frac{d}{d t} \delta \hat{q} & =\frac{1}{\Gamma\left[f^{\prime}(q)\right]^{2}}\left[-V^{\prime \prime}(q) \delta \hat{q}-2 \Gamma p f^{\prime}(q) f^{\prime \prime}(q) \delta \hat{q}\right] \\
& +\frac{1}{\Gamma\left[f^{\prime}(q)\right]^{2}}\left[\eta(t) f^{\prime \prime}(q) \delta \hat{q}\right]+O\left(\delta \hat{q}^{2}\right)
\end{aligned}
$$

With the help of Eq. (3.27) we then obtain the equations for $\left\langle\delta \hat{q}^{n}\right\rangle$

$$
\begin{aligned}
\frac{d}{d t}\left\langle\delta \hat{q}^{2}\right\rangle & =\frac{2}{\Gamma\left[f^{\prime}(q)\right]^{2}}\left[-V^{\prime \prime}(q)\left\langle\delta \hat{q}^{2}\right\rangle\right] \\
& -\frac{4}{\Gamma\left[f^{\prime}(q)\right]^{2}}\left[\Gamma p f^{\prime}(q) f^{\prime \prime}(q)\left\langle\delta \hat{q}^{2}\right\rangle\right] \\
& +\frac{2}{\Gamma\left[f^{\prime}(q)\right]^{2}}\left[\eta(t) f^{\prime \prime}(q)\left\langle\delta \hat{q}^{2}\right\rangle\right]+O\left(\left\langle\delta \hat{q}^{3}\right\rangle\right)
\end{aligned}
$$

$$
\begin{aligned}
\frac{d}{d t}\left\langle\delta \hat{q}^{3}\right\rangle & =\frac{3}{\Gamma\left[f^{\prime}(q)\right]^{3}}\left[-V^{\prime \prime}(q)\left\langle\delta \hat{q}^{3}\right\rangle\right] \\
& -\frac{6}{\Gamma\left[f^{\prime}(q)\right]^{3}}\left[\Gamma p f^{\prime}(q) f^{\prime \prime}(q)\left\langle\delta \hat{q}^{3}\right\rangle\right] \\
& +\frac{3}{\Gamma\left[f^{\prime}(q)\right]^{3}}\left[\eta(t) f^{\prime \prime}(q)\left\langle\delta \hat{q}^{3}\right\rangle\right]+O\left(\left\langle\delta \hat{q}^{4}\right\rangle\right)
\end{aligned}
$$

and so on. (It is apparent from Eqs. 3.28 3.29) that in the overdamped limit the higher order quantum contributions are small since each successive order of correction is lower than the preceding one by a factor of $\frac{1}{\Gamma}$ )

A simplified expression for the leading order quantum correction term $\left\langle\delta \hat{q}^{2}\right\rangle$ can be estimated by neglecting the higher order coupling terms in the square bracket in Eq. (3.28) (since the nonlinearity of the potential is small the terms of the order of $f^{\prime \prime}(x)$ are small) and rewriting it as $d\left\langle\delta \hat{q}^{2}\right\rangle=\frac{2}{\Gamma\left[f^{\prime}(q)\right]^{2}} V^{\prime \prime}(q)\left\langle\delta \hat{q}^{2}\right\rangle d t$. The overdamped deterministic mean motion on the other hand gives $d q=-\frac{V^{\prime}(q)}{\Gamma\left[f^{\prime}(q)\right]^{2}} d t$. These together yield after integration [29]

$$
\left\langle\delta \hat{q}^{2}\right\rangle=\Delta_{q}\left[V^{\prime}(q)\right]^{2}
$$

where $\Delta_{q}=\frac{\left\langle\delta \hat{q}^{2}\right\rangle_{0}}{\left[V^{\prime}\left(q_{0}\right)\right]^{2}}$ and $q_{0}$ is a quantum mechanical mean position at which $\left\langle\delta \hat{q}^{2}\right\rangle$ becomes minimum, i. e., $\left\langle\delta \hat{q}^{2}\right\rangle_{q_{0}}=\frac{1}{2} \hbar / \omega_{0}, \omega_{0}$ being defined earlier.

The relevant quantum correction terms (A1), A4) and (A5) can be rewritten for the potential (3.1) and coupling term (3.2) as follows,

$$
\begin{aligned}
Q_{V} & =-\frac{1}{2} V^{\prime \prime \prime}(q)\left\langle\delta \hat{q}^{2}\right\rangle, \\
Q_{f} & =0, \\
Q_{3} & =\left[f^{\prime \prime}(q)\right]^{2}\left\langle\delta \hat{q}^{2}\right\rangle .
\end{aligned}
$$

$V^{\prime \prime \prime \prime}(q)$ and $f^{\prime \prime \prime}(q)$ vanish for the chosen metastable potential and square-linear coupling. So by calculating the quantum dispersion term $\left\langle\delta \hat{q}^{2}\right\rangle$ we can find out the values of $\Delta_{1}, \Delta_{2}, \Delta_{v_{1}}, \Delta_{v_{2}}$ and $\Delta \phi$ as given by Eqs (3.17 3.18) and (3.21). The corrections (3.31) and (3.33) are reminiscent of the perturbation corrrections to Kramers escape rate due to color 43 . It may also be mentioned that important nonlinear terms are those represented by a linear chain coupled to a linear heat bath [44] and in spite of the different coupling Hamiltonian, the effects on the relevant rates are similar.

We now proceed to illustrate numerically the behavior of quantum transition rate given by Eq.(3.16). It appears that in addition to the barrier height the pre-factor is also affected by the quantum correction terms. The calculated quantum transition rate Eq.(3.16) implies that both the barrier height and the frequency factor contain the effects of quantum corrections due to nonlinearity of system potential and coupling function. The effect of quantization of the reservoir and quantum correction due 
to system nonlinearity is apparent in Fig.2 in the variation of $\ln k$ with $1 / T$ (Arrhenius plot) for varied contribution of nonlinear coupling. All the curves exhibit linearity at higher temperature and nonlinear variation is observed at lower temperature. Decrease in $b_{2}$ (squarelinear coupling contribution is proportional to $b_{2}$ ) results in enhancement of transition rate. This physically implies that with increased values of $b_{2}$ potential well becomes comparatively steeper (as shown in the Fig.1) and hence the transition rate decreases.

The effect of quantization of a classical transition rate is shown in Fig.3, where we make a comparison of $k$ vs $1 / T$ profiles for classical and quantum cases. One observes that at low temperature regime the classical transition rate is significantly lower in magnitude than quantum rate and at higher temperature the effect of quantization become insignificant. To clarify the effects of quantization we present also an Arrhenius plot comparing the classical and quantum regimes in the inset of Fig.3. From the inset plot it is clear that at the high temperature regime the plot exhibits linearity in the both cases, which is the standard Arrhenius classical result. In low temperature limit, however, one observes a much slower variation in the quantum case than that of classical one. To examine this in more detail it is not difficult to see that the exponential factor in the rate expression Eq.(3.16) can be reduced to a form containing the usual Arrhenius term exp $\left[-\left(\frac{\psi\left(q_{b}\right)-\psi\left(q_{a}\right)}{k T}\right)\right]$ times a $T^{2}$ enhancement term at low temperature of the form $\exp \left[\hbar \omega\left(\frac{\psi\left(q_{b}\right)-\psi\left(q_{a}\right)}{2(k T)^{2}}\right)\right]$. The full quantum behavior can be interpreted in terms of an interplay between the quantum diffusion coefficient $D_{q}$ and the quantum correction due to nonlinearity of the system potential appearing in $\Psi(q)$. When the temperature of the system is very low, i. e., in the vacuum limit or in the deep tunneling region the anharmonic terms in the potential do not contribute significantly. On the other hand as the temperature of the system increases significantly, $D_{q}$ increases resulting in decrease of the effective potential and hence $D_{q}$ and $Q$ (quantum correction terms) compete to cancel the effect of each other at higher temperature.

\section{CONCLUSION}

Based a theory of stochastic dynamics of a quantum particle in inhomogeneous media under overdamped condition we have calculated the rate of decay of a metastable state of the system under the influence of quantum state dependent friction and diffusion. It has been shown that the nonlinear interaction between the system and bath have its imprints on the pre-exponential factor as well as on the form of a generalized effective potential. Furthermore the nonlinearity of the effective potential can be identified as a typical consequence of quantum effect. The present theory can also be extended to other selected areas of chemical physics where the fluctuations in the potential in the system-reservoir coupling are important. From a purely quantum mechanical point of view such interactions give rise to multi-photon transition terms in the dynamics. Thus their contribution to phase relaxation in addition to population decay is of considerable significance. The difference between linear-linear and quadratic-linear coupling in the systemreservoir interactions terms is likely to be also important if one calculates the time evolution of flux-flux correlation functions [45] which in consequence gives rise to timedependent rate constant. The focus of the present article being the non-equilibrium steady state rate constant and ensuring a correct description of equilibrium in the quantum system which often remains vague in many related situations, we feel that an elaboration on this issue needs more detailed consideration. We note, in passing, that the treatment presented here concerns stochastic processes under overdamped condition in the Markovian limit. Extension of the theory to non-Markovian and weak friction regime is worth-pursuing in the context of rate theory and related contexts.

\section{Acknowledgments}

Thanks are due to the Council of Scientific and industrial research, Govt. of India, for partial financial support.

\section{APPENDIX A: QUANTUM CORRECTION TERMS}

Referring to the quantum nature of the system in the Heisenberg picture we now write the system operators $\hat{q}$ and $\hat{p}$ as $\hat{q}=q+\delta \hat{q}$ and $\hat{p}=p+\delta \hat{p}$ respectively. $\delta \hat{q}$ and $\delta \hat{p}$ represent quantum fluctuations around their respective mean values. By construction $\langle\delta \hat{q}\rangle=\langle\delta \hat{p}\rangle=0$ and they also follow the usual commutation relation $[\delta \hat{q}, \delta \hat{p}]=i \hbar$. Using a Taylor series expansion in $\delta \hat{q}$ around $q$ and $\delta \hat{p}$ around $p$ we express $Q_{V}, Q_{1}, Q_{2}$ and $Q_{3}, Q_{4}, Q_{5}$, and $Q_{f}$ as functions of $q, p,\left\langle\delta \hat{q}^{n}\right\rangle$ and $\left\langle\delta \hat{p}^{n}\right\rangle$ as follows;

$$
\begin{aligned}
Q_{V} & =V^{\prime}(q)-\left\langle V^{\prime}(\hat{q})\right\rangle \\
& =-\sum_{n \geq 2} \frac{1}{n !} V^{n+1}(q)\left\langle\delta \hat{q}^{n}\right\rangle \\
Q_{1} & =\Gamma\left[\left[f^{\prime}(q)\right]^{2} p-\left\langle\left[f^{\prime}(\hat{q})\right]^{2} \hat{p}\right\rangle\right] \\
& =-\Gamma\left[2 p f^{\prime}(q) Q_{f}+p Q_{3}+2 f^{\prime}(q) Q_{4}+Q_{5}[\mathrm{~A} 2)\right. \\
Q_{2} & =\eta(t)\left[\left\langle f^{\prime}(\hat{q})\right\rangle-f^{\prime}(q)\right] \\
& =\eta(t) Q_{f}
\end{aligned}
$$


where,

$$
\begin{aligned}
Q_{f} & =\sum_{n \geq 2} \frac{1}{n !} f^{n+1}(q)\left\langle\delta \hat{q}^{n}\right\rangle \\
Q_{3} & =\sum_{m \geq 1} \sum_{n \geq 1} \frac{1}{m !} \frac{1}{n !} f^{m+1}(q) f^{n+1}(q)\left\langle\delta \hat{q}^{m} \delta \hat{q}^{n}\right\rangle \\
Q_{4} & =\sum_{n \geq 1} \frac{1}{n !} f^{n+1}(q)\left\langle\delta \hat{q}^{n} \delta \hat{p}\right\rangle \\
Q_{5} & =\sum_{m \geq 1} \sum_{n \geq 1} \frac{1}{m !} \frac{1}{n !} f^{m+1}(q) f^{n+1}(q)\left\langle\delta \hat{q}^{m} \delta \hat{q}^{n} \delta \hat{p}\right\rangle
\end{aligned}
$$

The dynamics of these correction terms can be calculated [17] with the help of the following equations, which can be derived using the operator Langevin equations 2.6a and (2.6b) and by carrying out quantum mechanical average over the initial product separable coherent bath states.

$$
\begin{gathered}
\dot{\delta \hat{q}}=\delta \hat{p} \\
\dot{\delta \hat{p}}=-V^{\prime \prime}(q) \delta \hat{q}-\sum_{n \geq 2} \frac{1}{n !} V^{n+1}(q)\left[\delta \hat{q}^{n}-\left\langle\delta \hat{q}^{n}\right\rangle\right] \\
-2 \Gamma f^{\prime}(q) f^{\prime \prime}(q) \delta \hat{q}+2 \Gamma f^{\prime}(q) \sum_{n \geq 2} \frac{1}{n !} f^{n+1}(q)\left[\delta \hat{q}^{n}-\left\langle\delta \hat{q}^{n}\right\rangle\right. \\
+\sum_{m \geq 1} \sum_{n \geq 1} \frac{1}{m !} \frac{1}{n !} f^{m+1}(q) f^{n+1}(q)\left[\delta \hat{q}^{m} \delta \hat{q}^{n}-\left\langle\delta \hat{q}^{m} \delta \hat{q}^{n}\right\rangle\right] p \\
-\Gamma\left[f^{\prime}(q)\right]^{2} \delta \hat{p}+2 f^{\prime}(q) \sum_{n \geq 1} \frac{1}{n !} f^{n+1}(q)\left[\delta \hat{q}^{n} \delta \hat{p}-\left\langle\delta \hat{q}^{n} \delta \hat{p}\right\rangle\right] \\
+\sum_{m \geq 1} \sum_{n \geq 1} \frac{1}{m !} \frac{1}{n !} f^{m+1}(q) f^{n+1}(q)\left[\delta \hat{q}^{m} \delta \hat{q}^{n} \delta \hat{p}-\left\langle\delta \hat{q}^{m} \delta \hat{q}^{n} \delta \hat{p}\right\rangle\right] \\
+\eta(t)\left[f^{\prime \prime}(q) \delta \hat{q}+\sum_{n \geq 2} \frac{1}{n !} f^{n+1}(q)\left[\delta \hat{q}^{n}-\left\langle\delta \hat{q}^{n}\right\rangle\right]\right]
\end{gathered}
$$

The operator correction equations can be used to yield an infinite hierarchy of equations. Up to third order we construct, for example, the following set of equations which are coupled to quantum Langevin equations from (2.142.15;

$$
\begin{aligned}
& \frac{d}{d t}\left\langle\delta \hat{q}^{2}\right\rangle=\langle\delta \hat{q} \delta \hat{p}+\delta \hat{p} \delta \hat{q}\rangle \\
& \frac{d}{d t}\langle\delta \hat{q} \delta \hat{p}+\delta \hat{p} \delta \hat{q}\rangle=-2 \chi(q, p)\left\langle\delta \hat{q}^{2}\right\rangle+2\left\langle\delta \hat{q}^{2}\right\rangle \\
& -\Gamma\left[f^{\prime}(q)\right]^{2}\langle\delta \hat{q} \delta \hat{p}+\delta \hat{p} \delta \hat{q}\rangle-\zeta(q, p)\left\langle\delta \hat{q}^{3}\right\rangle \\
& \text { - } 2 \Gamma f^{\prime}(q) f^{\prime \prime}(q)\left\langle\delta \hat{q}^{2} \delta \hat{p}+\delta \hat{p} \delta \hat{q}^{2}\right\rangle \\
& \frac{d}{d t}\left\langle\delta \hat{p}^{2}\right\rangle=-2 \Gamma\left[f^{\prime}(q)\right]^{2}\left\langle\delta \hat{p}^{2}\right\rangle-\chi(q, p)\langle\delta \hat{q} \delta \hat{p}+\delta \hat{p} \delta \hat{q}\rangle \\
& -\frac{1}{2} \zeta(q, p)\left\langle\delta \hat{q}^{2} \delta \hat{p}+\delta \hat{p} \delta \hat{q}^{2}\right\rangle \\
& -2 \Gamma f^{\prime}(q) f^{\prime \prime}(q)\left\langle\delta \hat{q} \delta \hat{p}^{2}+\delta \hat{p}^{2} \delta \hat{q}\right\rangle \\
& \frac{d}{d t}\left\langle\delta \hat{q}^{3}\right\rangle=\frac{3}{2}\left\langle\delta \hat{q}^{2} \delta \hat{p}+\delta \hat{p} \delta \hat{q}^{2}\right\rangle \\
& \frac{d}{d t}\left\langle\delta \hat{p}^{3}\right\rangle=-3 \Gamma\left[f^{\prime}(q)\right]^{2}\left\langle\delta \hat{p}^{3}\right\rangle \\
& -\frac{3}{2} \chi(q, p)\left\langle\delta \hat{q} \delta \hat{p}^{2}+\delta \hat{p}^{2} \delta \hat{q}\right\rangle \\
& \frac{d}{d t}\left\langle\delta \hat{q}^{2} \delta \hat{p}+\delta \hat{p} \delta \hat{q}^{2}\right\rangle=-2 \chi(q, p)\left\langle\delta \hat{q}^{3}\right\rangle+2\left\langle\delta \hat{q} \delta \hat{p}^{2}+\delta \hat{p}^{2} \delta \hat{q}\right\rangle \\
& -\Gamma\left[f^{\prime}(q)\right]^{2}\left\langle\delta \hat{q}^{2} \delta \hat{p}+\delta \hat{p} \delta \hat{q}^{2}\right\rangle \\
& \frac{d}{d t}\left\langle\delta \hat{q} \delta \hat{p}^{2}+\delta \hat{p}^{2} \delta \hat{q}\right\rangle=2\left\langle\delta \hat{p}^{3}\right\rangle-4 \chi(q, p)\left\langle\delta \hat{q}^{2} \delta \hat{p}+\delta \hat{p} \delta \hat{q}^{2}\right\rangle \\
& -2 \Gamma\left[f^{\prime}(q)\right]^{2}\left\langle\delta \hat{q} \delta \hat{p}^{2}+\delta \hat{p}^{2} \delta \hat{q}\right\rangle \\
& \chi(q, p)=V^{\prime \prime}(q)+2 \Gamma p f^{\prime}(q) f^{\prime \prime}(q)-\eta(t) f^{\prime \prime}(q) \\
& \zeta(q, p)=V^{\prime \prime \prime}(q)+2 \Gamma p f^{\prime}(q) f^{\prime \prime \prime}(q) \\
& +2 \Gamma p\left[f^{\prime \prime}(q)\right]^{2}-\eta(t) f^{\prime \prime \prime}(q)
\end{aligned}
$$

[1] R. Zwanzig, Lectures in Theoretical Physics, Vol.3; J. Stat. Phys. 9, 215 (1973).

[2] A. O. Caldeira and A. J. Leggett, Ann. Phys. (N.Y) 149, 374 (1983).

[3] A. O. Caldeira and A. J. Leggett, Ann. Phys. (N.Y) 153, 445 (1984).

[4] H. Grabert, P. Schramm and G. L. Ingold, Phys. Rep. 168, 115 (1988).

[5] K. Okumura and Y. Tanimura, Phys. Rev. E 56, 2747 (1997); T. Steffen and Y. Tanimura, J. Phys. Soc. Jap. 69, 3115; T. Kato and Y. Tanimura, J. Chem. Phys. 117, 6221 (2002); ibid. 120, 260 (2004).

[6] D. Barik, D. Banerjee and D. S. Ray, in Progress in
Chemical Physics Research, Vol- 1, Edited by A. N. Linke, (Nova Publishers, New York, ISBN: 1-59454-4514, 2006).

[7] U. Weiss, Quantum Dissipative systems (World Scientific, Singapore, 1999).

[8] J. M. Sancho, M. San Miguel, S. L. Katz and J. D. Gunton, Phys. Rev. A 26, 1589 (1982).

[9] J. M. Sancho, M. San Miguel and D. Dür, J. Stat. Phys. 28, 291 (1982).

[10] A. M. Jayannavar and M. C. Mahato, Pramana 45, 368 (1995).

[11] A. Hernandez-Machado, M. SanMiguel and J. M. Sancho, Phys. Rev. A 29, 3388 (1984). 
[12] J. Masoliver and L. Garrido, Phys. Lett. A 103, 366 (1984)

[13] J. D. Ramshaw and K. Lindenberg, J. Stat. Phys. 45, 295 (1986).

[14] H. Sakaguchi, J. Phys. Soc. Jpn. 70, 3247 (2001).

[15] C. Anteneodo and C. Tsallis, J. Math. Phys. 44, 5194 (2003).

[16] J. Garcia-Ojalvo and J. M. Sancho, Noise in spatially extended systems (Springer-Verlag, New York, 1999).

[17] D. Barik and D. S. Ray, J. Stat. Phys. 120, 339 (2005).

[18] W. H. Miller, S. D. Schwartz and J. W. Tromp, J. Chem. Phys. 79, 4889 (1983); P. Hänggi, P. Talkner and M. Borkoves, Rev. Mod. Phys., 62, 251(1990).

[19] M. Boguñá, J. M. Porrá, J. Masoliver and K. Lindenberg, Phys. Rev. E 57, 003990 (1998).

[20] D. E. Makarov and M. Topaler Phys. Rev. E 52, 178 (1995).

[21] J. D. Bao, Phys. Rev. A 65, 052120 (2002); J. D. Bao, Phys. Rev. A 69, 022102 (2004).

[22] P. K. Ghosh, D. Barik, B. C. Bag and D. S. Ray, J. Chem. Phys. 123, 224104 (2005).

[23] M. Büttiker, Z. Phys. B: Condensed Matter 68, 161 (1987).

[24] P. Reimann, Phys. Rep. 361, 57 (2002).

[25] S. Faetti, P. Grigolini and F. Marchesoni, Z. Phys. B 47, 353 (1982).

[26] R. Benzi, G. Parisi, A. Sutera, and A. Vulpiani, Tellus 34, 10 (1982); B. McNamara, K. Wiesenfeld and R. Roy, Phys. Rev. Lett. 60, 2626 (1988).

[27] L. Gammaitoni, F. Marchesoni, E. Menichella-Saetta and S. Santucci, Phys. Rev. Lett. 62, 349 (1989); L. Gammaitoni, F. Marchesoni, E. Menichella -Saetta, and S. Santucci, Phys. Rev. E 49, 4878 (1994); L. Gammaitoni, P. Hänggi, P. Jung and F.Marchesoni, Rev. Mod. Phys., 70, 223(1998).

[28] W. Horsthemke and R. Lefever, Noise-induced transitions: Theory and applications in physics, chemistry, and biology (Springer-Verlag, Berlin and New York, 1984).

[29] P. K. Ghosh, D. Barik and D. S. Ray, Phys. Lett. A 342 12 (2005).

[30] S. Mukamel, Principles of Nonlinear Optical Spectroscopy (Oxford University Press, New York, 1995).

[31] D. W. Oxtoby, Adv. Chem. Phys 40, 1 (1979).

[32] J. S. Bader and B. J. Berne, J. Chem. Phys. 100, 8359 (1994).

[33] W. T. Pollard and R. A. Friesner, J. Chem. Phys. 100, 5054 (1994).

[34] E. P. Wigner, Phys. Rev. 40, 749 (1932); M. Hillery, R.
F. O'Connell, M. O. Scully and E. P. Wigner, Phys. Rep. 106, 121 (1984).

[35] K. Lindenberg and V. Seshadri, Physica A, 109, 483 (1981); K. Lindenberg and E. Cortés, ibid. 126, 489 (1984).

[36] W. H. Louisell, Quantum Statistical Properties of Radiation (J. Wiley, 1973).

[37] S. K. Banik, B. C. Bag and D. S. Ray, Phys. Rev. E 65, 051106 (2002).

[38] P. K. Ghosh, D. Barik and D. S. Ray, Phys. Rev. E 71, 041107 (2005); D. Banerjee, B. C. Bag, S. K. Banik and D. S. Ray, J. Chem. Phys. 120, 8960 (2004)

[39] D. Barik, S. K. Banik and D. S. Ray, J. Chem. Phys. 119, 680 (2003); D. Barik, B. C. Bag and D.S. Ray, J. Chem. Phys. 119, 12973 (2003).

[40] D. Barik and D. S. Ray, J. Chem. Phys. 121, 1681 (2004).

[41] R. Landauer, Phys. Rev. A 12, 636 (1975).

[42] R. Landauer, J. Stat. Phys. 53, 233 (1988).

[43] P. Hänggi, F. Marchesoni and P. Grigolini, Z. Phys. B 56, 333 (1984)

[44] P. Hänggi, F. Marchesoni and P. Sodano, Phys. Rev. Lett. 60 , 2563 (1988); P. Hänggi, F. Marchesoni and P. Riseborough Europhys. Lett. 13, 217 (1990).

[45] J. B. Straus and G. A. Voth, J. Chem. Phys. 96,5460 (1991); J. B. Straus, J. M. Liorente, and G. A. Voth, J. Chem. Phys. 98,4082 (1991); G. A. Voth, J. Chem. Phys. 975908 (1993).

\section{Figure Captions}

Fig.1: Schematic illustration of the effect of linearlinear(a) and square-linear(b) system-bath coupling on a relevant potential system.

Fig.2: Plot of $\ln k$ vs. $1 / T$ for different strength of nonlinear coupling $b_{2}\left((\mathrm{i}) b_{2}=0.01\right.$ (dashed line) (ii) $b_{2}=$ 0.1 (solid line) (iii) $b_{2}=0.2$ (dotted line) for a fixed parameter set $a=0.5, b=0.15, b_{1}=1.0$ and $\Gamma=1.0$ (all the quantities are in dimensionless unit).

Fig.3: A comparison between classical(solid line) and quantum (dotted line) decay rate plotting $k$ as a function of $1 / T$ for the parameter set $a=0.5, b=0.15, b_{1}=$ $1.0, b_{2}=0.10$ and $\Gamma=1.0$. The inset presents a variation of $\ln k$ with $1 / T$ comparing classical (solid line) and quantum (dotted line) cases for the same parameter set as in the main figure.(all the quantities are in dimensionless unit). 


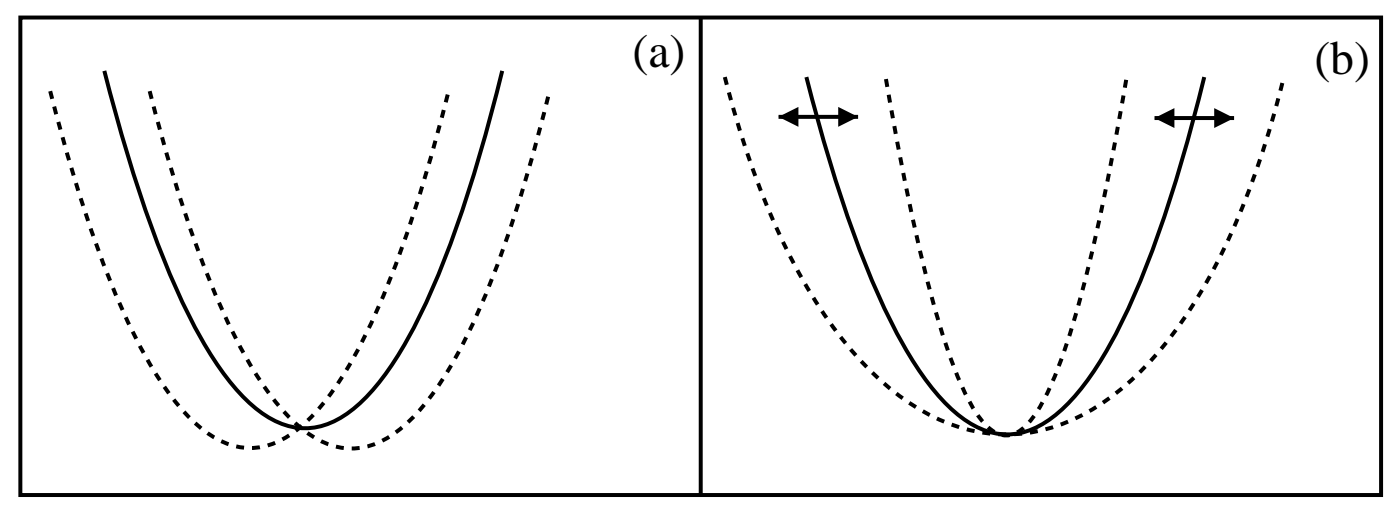

Fig.1 


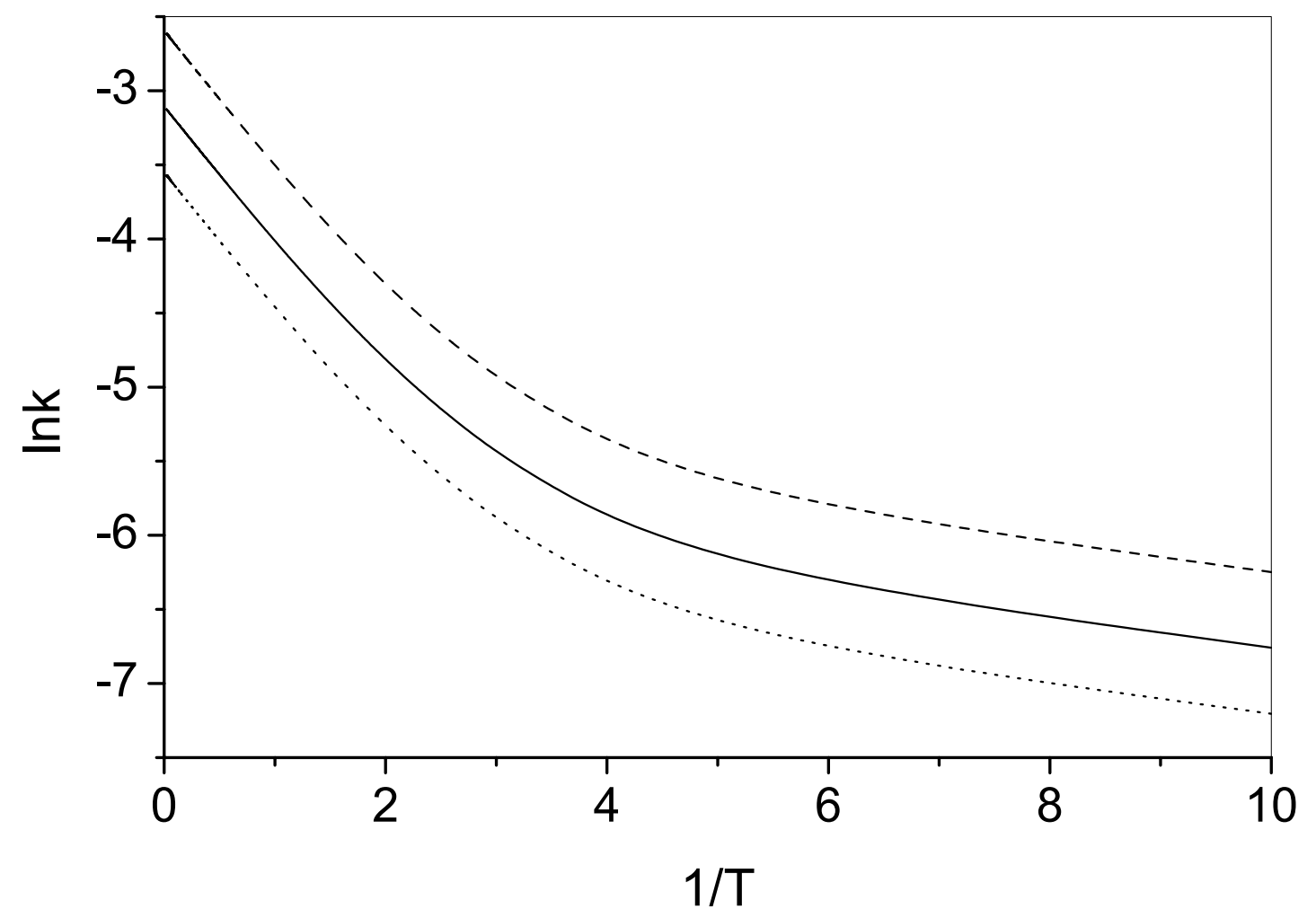

Fig.2 


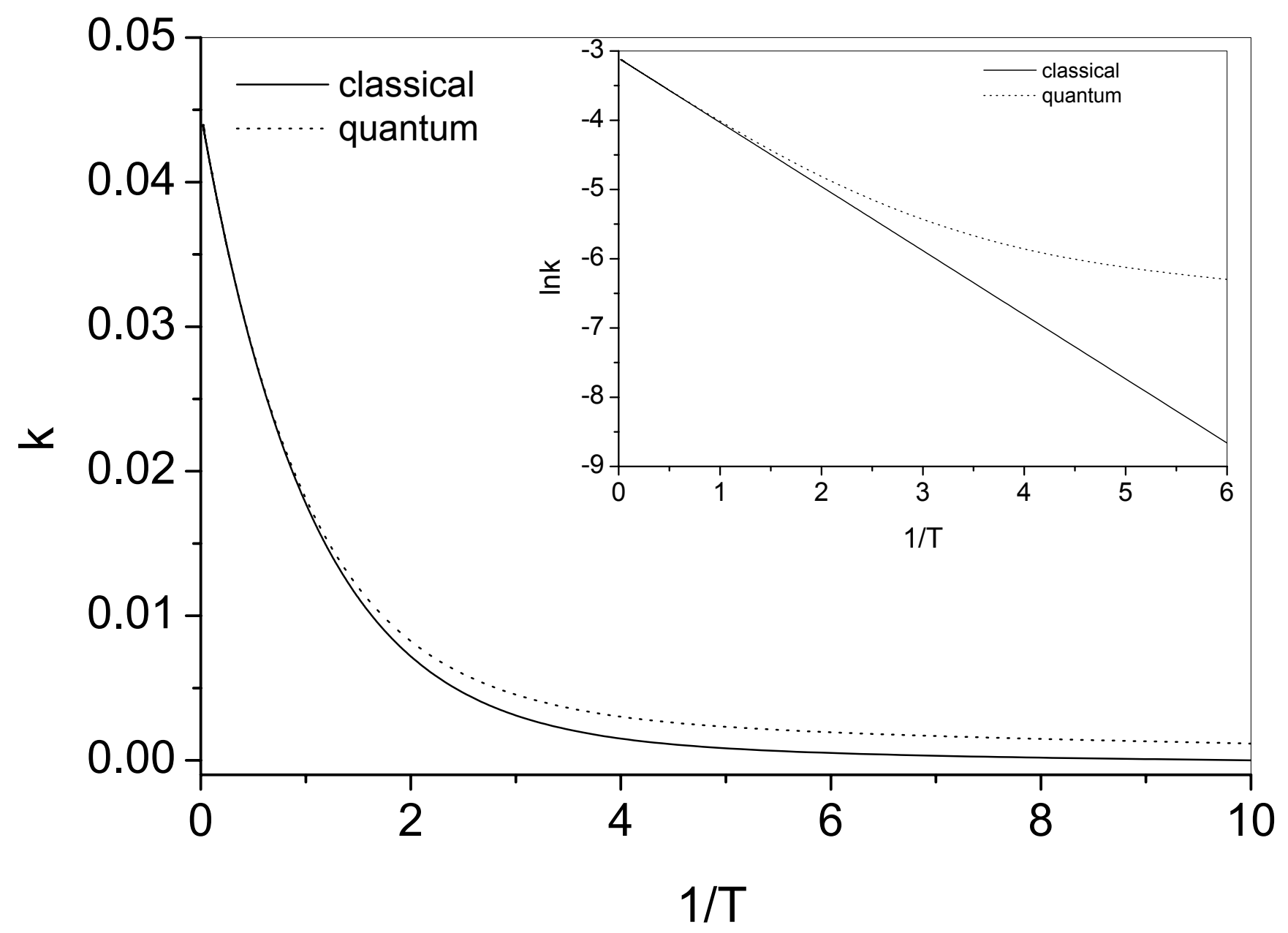

Fig.3 